\title{
Understanding pop-ins in spherical nanoindentation
}

\author{
Siddhartha Pathak, ${ }^{1, a)}$ Jessica L. Riesterer, ${ }^{2, b)}$ Surya R. Kalidindi, ${ }^{3}$ and Johann Michler ${ }^{2}$ \\ ${ }^{1}$ MPA-CINT Center for Integrated Nanotechnologies, Los Alamos National Laboratory, Los Alamos, \\ New Mexico 87545, USA \\ ${ }^{2}$ Empa, Swiss Federal Laboratory for Materials Testing and Research, Laboratory for Mechanics of Materials \\ and Nanostructures, Feuerwerkerstrasse 39, Thun 3602, Switzerland \\ ${ }^{3}$ George W. Woodruff School of Mechanical Engineering, Georgia Institute of Technology, Atlanta, \\ Georgia 30332, USA
}

(Received 19 August 2014; accepted 8 October 2014; published online 24 October 2014)

\begin{abstract}
Pop-ins, or sudden displacement-bursts at constant load in a nanoindentation test, are typically attributed to the difficulty of setting up potent dislocation sources in the very small indentation zones in these experiments. Such displacement (and strain) bursts would intuitively indicate a sharp drop in stress during the pop-in event itself. However, spherical indentation stress-strain curves routinely exhibit a high and stable indentation stress value during the pop-in, and the indentation stresses decrease only after a further finite amount of additional indentation displacement has been applied. In order to understand this discrepancy, we utilize a combination of interrupted spherical indentation tests along with depth profiling of the residual indentation surfaces using in-situ atomic force microscopy (AFM) to study pop-ins. The AFM surface profile maps show that there is an asymmetric profile change over a limited region around the indentation contact area for a single pop-in; the asymmetry disappears upon further loading beyond the pop-in. A plausible sequence of physical processes (related to metal plasticity) occurring underneath the indenter during and immediately after the occurrence of the pop-in is proposed to explain these observations. (C) 2014 AIP Publishing LLC. [http://dx.doi.org/10.1063/1.4898698]
\end{abstract}

Since bulk crystals inherently contain preexisting flaws and defects in the form of dislocations, they rarely exhibit near-theoretical stresses. In this respect, submicrometer volume contact experiments, where the exceedingly small volume probed is potentially defectfree, offer a unique opportunity to explore the material response under very high stresses. During nanoindentation experiments using small radii spherical indenters (or sharp indenters with a residual radius at their tip), annealed crystalline materials have been observed to sustain extremely high stresses locally - the magnitude of these stresses sometimes approaching the theoretical shear strength of the material. ${ }^{1}$ After experiencing these very high stresses, these tests produce "pop-in" events that are generally characterized by sudden excursions in depth (for a load controlled experiment) which acts as a trigger for the onset of plastic deformation ${ }^{2}$ (Fig. 1). These pop-ins and their underlying mechanisms can be analyzed in significant detail with the help of our recently developed data analyses procedures for spherical nanoindentation, which transform the entire load-displacement dataset, including the initial loading segment containing the pop-ins, into much more meaningful indentation stress-strain (ISS) curves. As shown in this letter, these ISS curves can offer unique insights into the details of the pop-in events, where the causative mechanisms of incipient plasticity are substantially different and are averaged out in conventional macro-scale uniaxial tests. This improved understanding is critical if we are to extract meaningful parameters from

\footnotetext{
a) Electronic addresses: pathak@lanl.gov and siddharthapathak@gmail.com. Telephone: +1 (505) 667-2082. Fax: +1 (505) 665-9030.

b) Present address: FEI Company, Hillsboro, Oregon 97124, USA.
}

spherical indentation test protocols for quantitative description of metal plasticity at the very small length scales where pop-ins are expected to occur commonly. The main goals of this study were to carefully document the sequence of physical processes (related to metal plasticity) occurring underneath the indenter during and immediately after the occurrence of the pop-in. To this end, we have employed a combination of interrupted spherical indentation tests along with depth profiling of the residual indentation surfaces using atomic force microscopy (AFM).

Although there exists a large body of literature dedicated to the initiation of pop-ins and plastic yield at the nanoscale, ${ }^{3-6}$ very little is known about the events happening during the process itself. It is known that the pop-ins offer an unambiguous transition from reversible (predominantly elastic) to irreversible plastic deformation during the earliest stages of mechanical contact in indentation experiments. ${ }^{5}$ The magnitude of the local stress that produces this incipient plasticity in nano-scale contacts has been estimated to be close to the theoretical limit of the material. ${ }^{4,7}$ As noted before, elastic stresses of this magnitude are seldom achieved under macroscale testing conditions; only special cases such as single crystal metal whiskers, free of intrinsic defects, are known to demonstrate similar strengths under homogenous mechanical loading. ${ }^{8}$ Various other kinds of pop-ins have also been reported in indentation (e.g., cracks in brittle ceramics, ${ }^{9}$ shear bands in metallic glasses, ${ }^{10}$ interactions between moving dislocation and solute atoms, ${ }^{11}$ phase transformations, ${ }^{12}$ or "pop-outs" during unloading in $\mathrm{Si}^{13}$ ), which have very different physical origins. In this work, we focus only on the pop-ins related to incipient plasticity in the material. 

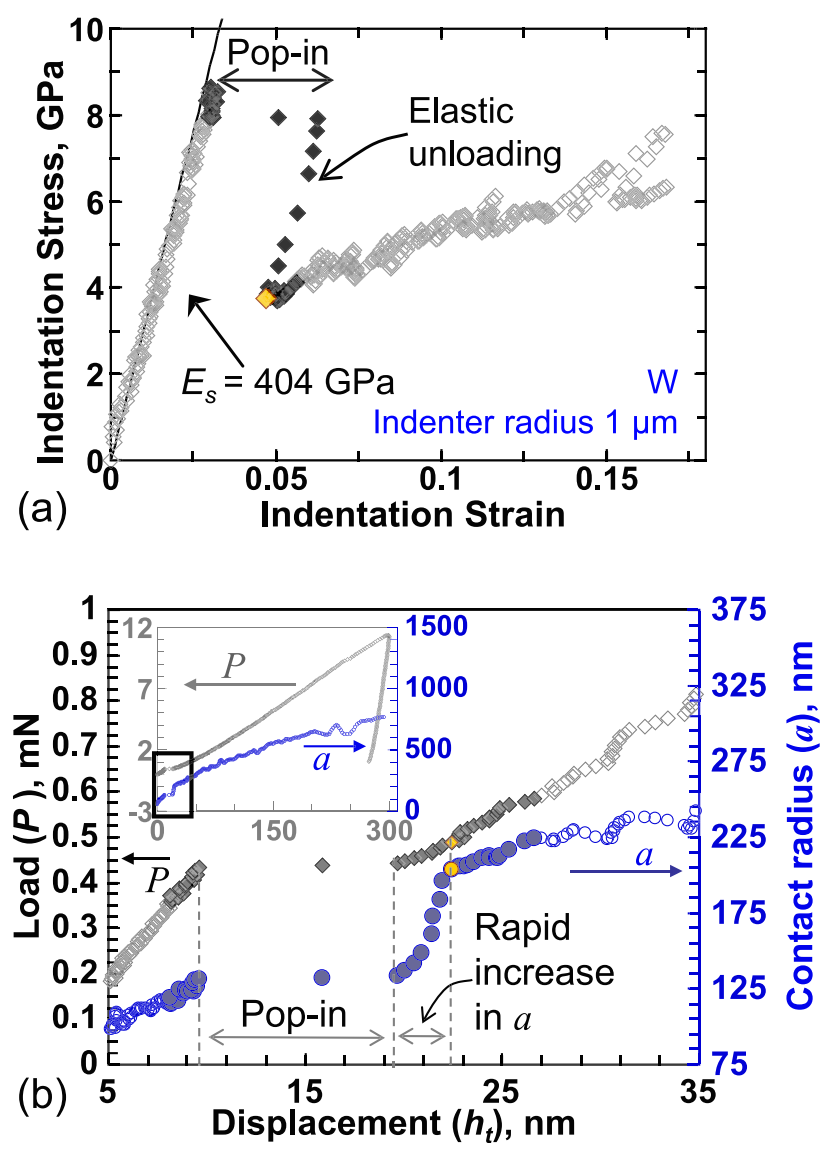

FIG. 1. (a) ISS response of a pop-in event in an electropolished tungsten sample using a spherical indenter of $1 \mu \mathrm{m}$ radius. The pop-in is manifested as a strain burst at constant stress, which is immediately followed by an unloading segment after the pop-in. (b) Corresponding measurements of the indentation load, displacement, and contact radius (CSM signal) show that the contact radius remains constant during the pop-in but increases rapidly immediately afterwards. Note the data point marked in gold color, which signifies the end of the regime of rapid increase in the contact radius, and its corresponding location in the ISS curve.

Pop-ins are revealed as sudden excursions in depth and strain (in a load controlled experiment) and have been generally attributed to the difficulty of setting up potent dislocation sources (e.g., Frank-Read sources ${ }^{14}$ ) in the very small indentation zones (typically much smaller than the length scales associated with dislocation spacing or dislocation cell size, see the illustration in Fig. $\mathrm{S}^{15}$ ) in these experiments. This physical explanation is consistent with the observations that the pop-ins occur most readily in indentation experiments on annealed samples with very small indenter tip radii. ${ }^{6,16}$ Their occurrence is more stochastic when using a larger indenter (Fig. S1(b) ${ }^{15}$ ), and pop-ins were almost absent in all of our indentation measurements on coldworked samples (Fig. S1(c)). ${ }^{15,17}$ Further evidence for this explanation came from the observations that pop-ins could be avoided by altering the sample surface with a small degree of mechanical polishing (such as when using vibratory polishing methods Fig. S1(d) ${ }^{15}$ ) presumably because it induces a minimal amount of dislocations in the sample surface layer, and the indentation stress-strain curves after the pop-in event on electro-polished samples seemed to approach the indentation stress-strain curves obtained on the vibro-polished sample without the pop-in (Fig. S1(d)). ${ }^{15,18}$
Figure 1(a) shows the typical ISS response of an indentation pop-in event for a $1 \mu \mathrm{m}$ radius spherical indenter on electro-polished samples of annealed W. Similar pop-ins have also been observed in a broad range of materials, including $\mathrm{Ag}$ (Fig. S3), $\mathrm{Al}, \mathrm{Au}, \mathrm{Cu},{ }^{17-20}$ and $\mathrm{Fe}-3 \% \mathrm{Si}$ (Fig. S2) ${ }^{15,19}$ and have consistently exhibited the following salient features in the ISS curves: (i) During the pop-in event, the indentation stress values remain high and stable, while there is a burst in the value of the indentation strain. (ii) Subsequent to the pop-in event, the indentation stress decreases only after a finite amount of additional indentation displacement has been applied. (iii) During this additional indentation displacement, the indentation strain actually decreases producing an unloading segment in the indentation stress-strain curve. (iv) The unloading modulus during this additional indentation displacement (subsequent to pop-in) is generally very close to the indentation modulus (see Figs. 1(a), S2(b), and $S 3(b)^{15}$ ) measured in the initial loading segment (except when the pop-ins are unusually large, ${ }^{18}$ Figs. S2(b) and $\left.\mathrm{S} 3(\mathrm{~b})^{15}\right)$.

Some of the above observations are rather perplexing since they differ from the reported literature. For example, an analysis based solely on the load and displacement signals (without using the continuous stiffness measurement (CSM) signal described below) would intuitively indicate a sharp drop in stress during the pop-in event itself. Similarly, other ISS protocols employed in literature have produced unloading segments with negative slopes ${ }^{21}$ for pop-ins, instead of a positive unloading slope shown in Fig. 1. These differences are explained below.

Figure 1(b) shows the corresponding load-contact radius-displacement $\left(P-a-h_{t}\right)$ data for the ISS pop-in curve shown in Fig. 1(a). A salient feature of the protocols used in Fig. 1 to extract the ISS curve from the $P-a-h_{t}$ data is the use of CSM signal ${ }^{22}$ for obtaining a reliable estimate of the radius of contact, $a$, at every datapoint (Eqs. (S1) and $\left.(\mathrm{S} 2)^{15,23}\right)$. Here, $a=S / 2 E_{e f f}$, where $S\left(=d P / d h_{e}\right)$ is the elastic stiffness from CSM and $E_{\text {eff }}$ is the effective modulus of the indenter and the specimen system. The rigorous derivation of $a$ from Eq. (S2) ${ }^{15}$ based directly on Hertz theory makes the estimates of contact radius from the measured CSM signals highly trustworthy. Each data point in Fig. 1 corresponds to one measurement in the experiment. Since the data acquisition rate was constant $(5 \mathrm{~Hz})$, subsequent points that are farther apart indicate a faster response compared to those that are closer to each other. For example, in the measurement shown in Fig. 1(b), there is only one intermediate measurement during the entire pop-in event (not counting the endpoints). This indicates that the pop-in event was very fast (on a much smaller time scale) compared to the rest of the loading history.

As expected, the load remains constant during the pop-in event in Fig. 1(b) (the measurements were conducted in load control) and consequently there is a significant excursion in the indentation depth. The surprising observation in Fig. 1(b) is that the estimated value of the contact radius (a) is also more or less constant during the pop-in event. Since $a$ is estimated directly from the CSM signal ( $a=S / 2 E_{\text {eff }}$, Eq. $(\mathrm{S} 2)^{15}$ ), the above observation reflects the fact that the stiffness measured by the machine just before and immediately 
after the pop-in is almost the same. This also explains the shape of the ISS curve shown in Fig. 1(a); since the load and the contact radius are essentially constant during the pop-in, the indentation stress is also almost constant during this event. The excursion in indentation depth produces an excursion in the indentation strain. Therefore, it is not surprising anymore that the indentation stress remains high and almost constant during the pop-in event.

The notion that the contact radius remains mostly constant during a pop-in is counter-intuitive as one would usually expect a sudden increase in the contact radius along with the large excursion in the indentation depth. However, if the contact radius would increase significantly during pop-in, we should definitely expect to see a corresponding change in the CSM signal. We have repeated measurements of the kind shown in Fig. 1 in a large number of annealed metal samples (including $\mathrm{W}, \mathrm{Fe}-3 \% \mathrm{Si}, \mathrm{Al}, \mathrm{Cu}$, and $\mathrm{Ag},{ }^{17-20}$ see Figs. $\mathrm{S} 1-\mathrm{S} 3^{15}$ ) and have consistently observed the same features. In all of our measurements, the contact radius appears to remain more or less constant during the pop-in event itself.

A second salient feature in the plots shown in Fig. 1 is that there is a short regime of rapid increase in $a$ (also seen in the CSM signal) with additional indentation depth immediately following the pop-in. Consequently, there is a rapid decrease in the indentation stress in this regime, which in turn is responsible for the unloading segment seen in the ISS curves. The data point at the end of this regime of rapid increase in the contact radius is marked with gold color in Fig. 1. By tracking the location of this particular data point in the different plots, it is clearly seen that this regime ends when the indentation stress has fallen to the levels consistent with ISS curves without pop-ins.

Interestingly, the unloading slope after pop-in in the ISS curve in Fig. 1 is very close to the slope in the initial elastic loading segment of the ISS curve. This is generally true when the pop-ins are small in size. Larger pop-ins reach higher stress levels (Fig. S2(b) ${ }^{15}$ ) and produce larger excursions in strain, presumably because they need to dissipate more energy before reaching the stress levels associated with ISS curves without pop-ins. They also show a much more rapid increase in contact radius after the pop-in event, and the slope of the unloading segment in the ISS curve immediately following the pop-in is no longer the same as the slope of the initial elastic loading segment (Fig. S2(b) ${ }^{15}$ ).

It is emphasized here that only the ISS curves produced using the data analyses protocols shown in Eqs. (S1) and $(\mathrm{S} 2)^{15}$ produce unloading slopes with positive slopes. Other ISS protocols reported in literature typically produce unloading segments with negative slopes ${ }^{21}$ for pop-ins. Unloading segments in the ISS curves with negative slopes are, in our opinion, completely unphysical as they would imply that, while the stress is reducing (which has to be accompanied by a reduction of elastic strain), the total strain is simultaneously increasing by significant amounts.

Figure 2 presents a conceptual model for what might happen during a pop-in that is consistent with the observations made above. As mentioned earlier, the pop-ins described in this study are produced by sudden activation of dislocation sources (upon reaching a certain combination of

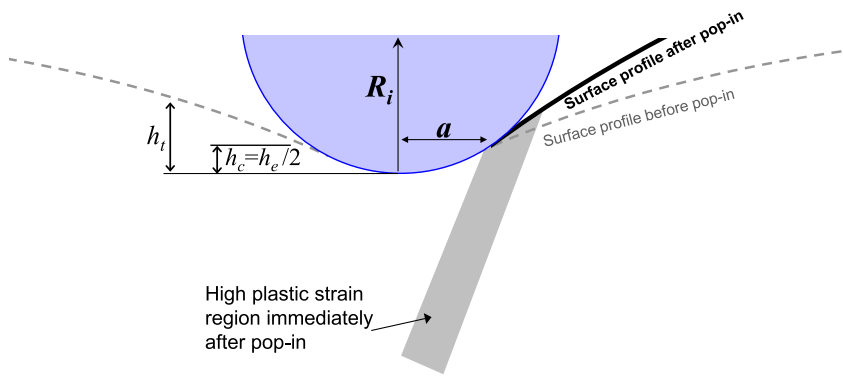

FIG. 2. Schematic description of the sequence of physical processes immediately following a pop-in in spherical nanoindentation. Before pop-in, the indentation response is predominantly elastic, and the sample surface profile (the curve in dotted gray) can be estimated from Hertz theory where $h_{c}=h_{e} / 2=h_{t} / 2$. Upon pop-in, there is a sudden increase in the indentation depth, but no immediate increase in $a$. Rather, the high plastic strain causes the dislocations to travel (in bursts) along specific crystallographic planes up to the free surface adjacent to the indenter. This brings the sample surface in closer proximity to the indenter (the curve in solid black on the right hand side of the contact region). Thus, any further loading causes a large increase in $a$.

local stress and size of the indentation zone), which in turn facilitates plastic strain under the indenter by the familiar process of dislocation slip on specific slip systems. ${ }^{4,7}$ The observations described in Fig. 1 suggest that the slip bursts that occur during the pop-in event are actually altering the profile of the sample surface without changing the contact radius significantly. These uncontrolled slip bursts help to diffuse the high elastic stresses under the indenter. It is well known from Hertz's model that the stress fields in the indentation zone are such that the highest driving force for plastic deformation occurs at some distance below the indenter tip (not on the sample surface). For an isotropic material, the highest shear stress (needed to initiate plastic strain) is expected to occur at a distance of $0.5 a$ below the indenter, where $a$ is the contact radius. Dislocations should therefore be expected to start well below the indenter tip and travel (in bursts) on specific crystallographic slip systems (e.g., [110] directions on (111) planes in fcc metals ${ }^{24}$ ). It is also important to recognize that the sample free surface is likely to play an important role in facilitating these slip bursts. For example, the rigid conformation of the indenter and the sample in the contact region would not allow the formation of the steplike features needed to release the dislocations. The sample free surface on the other hand will allow the easy release of the dislocations.

Figure 2 shows schematically how the dislocation burst of a pop-in might significantly alter the profile of the free surface of the sample just outside the indenter contact region, while not changing the contact radius itself. Such a process is expected to be asymmetric, i.e., the profile change is expected to happen only over a limited area for a single pop-in. However, any additional indentation depth immediately following the pop-in would cause a sharp increase in the contact radius (and a corresponding increase in the stiffness measurement). This expected rapid increase in contact radius and stiffness immediately after the pop-in matches well with the contact radius (CSM) measurements shown in Fig. 1.

While there is no easy way to provide direct evidence of these processes occurring under sub-micron volume contact 

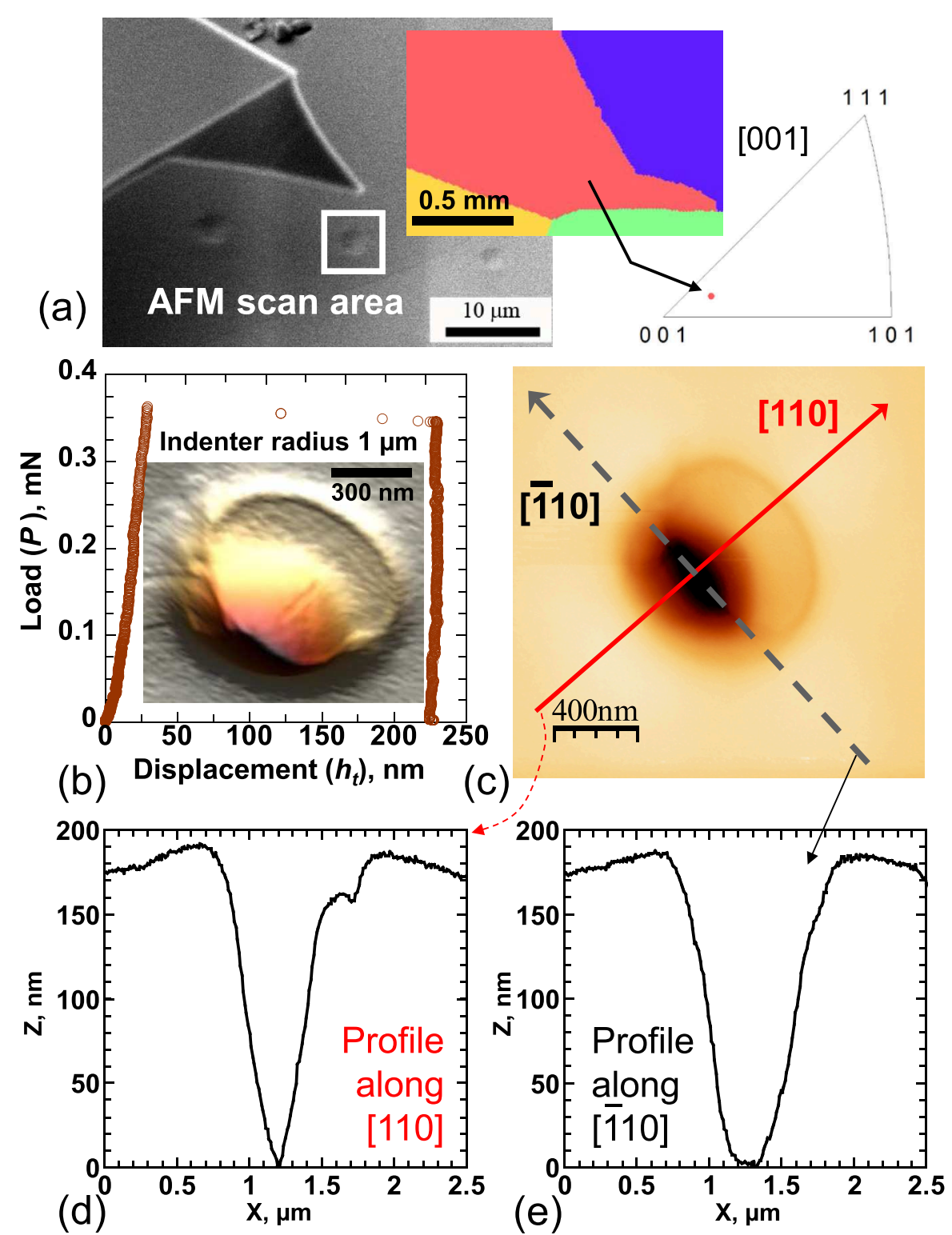

FIG. 3. (a) Test set-up of a custom built SPECS hybrid AFM inside a Tescan Lyra FIB/SEM operating in intermittentcontact mode. AFM measurements were done in the near-(001) red grain of an annealed electro-polished Al sample. (b) Load-displacement response of an indentation test stopped immediately after a single large $(\sim 180 \mathrm{~nm})$ pop-in event using a $1 \mu \mathrm{m}$ indenter. (Inset) AFM 3D and (c) 2D surface profiles of the residual imprint. (d) The line profiles show the formation of a large uplifted region inside the indent-imprint along the [110] direction of the sample surface. Note the anisotropy of the residual indent imprint; no such uplift is visible on the (e) perpendicular $[\overline{1} 10]$ direction.

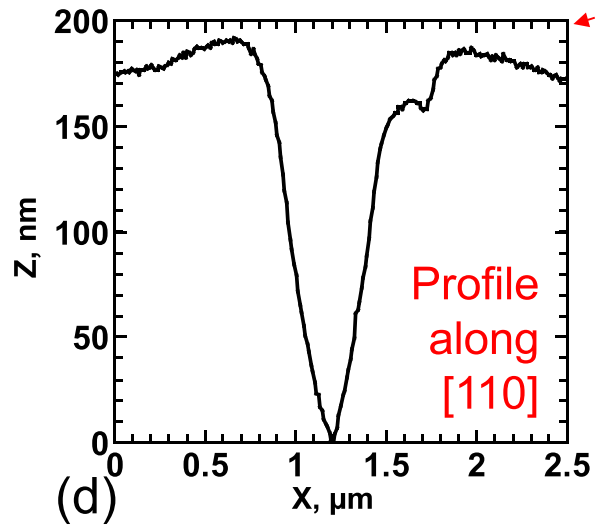

regions under the indenter, some indirect evidence can be obtained from post pop-in AFM measurements of the indents. For this purpose, we utilized a custom built SPECS hybrid AFM inside a Tescan Lyra FIB/SEM working in intermittentcontact mode to map the resultant surface profile immediately

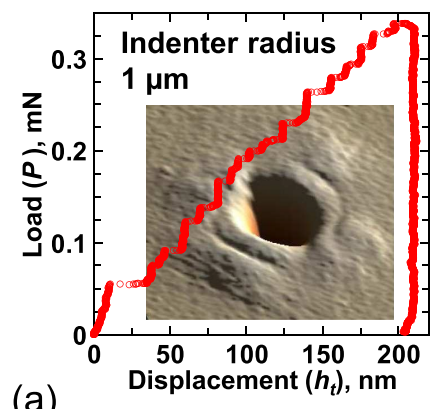

(a)

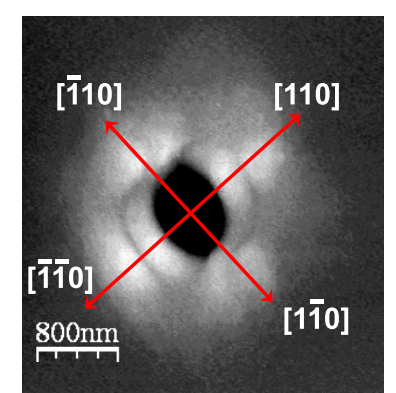

(b)
FIG. 4. (a) Load-displacement response on the same $\mathrm{Al}$ surface showing an indentation test with multiple pop-in events for a $1 \mu \mathrm{m}$ indenter. The (inset) AFM 3D and (b) 2D surface profiles of the residual imprint show a four-fold pattern of material pile-up around the indent, which is typical for a near(001) FCC orientation. after a pop-in event (after the load has been taken off). The utility of the in-situ setup is evident in Fig. 3(a) where the AFM needle can be seen poised over one of the indents in the SEM image; these indents can be notoriously difficult to find without such an arrangement. Indents with a $1 \mu \mathrm{m}$ spherical indenter were performed on a near (001) grain of annealed and electro-polished (inset of Fig. 3(a)). Responses similar to those described below in Figs. 3 and 4 were also seen for other orientations of $\mathrm{Al}$ as well as annealed and electropolished $\mathrm{Fe}-3 \% \mathrm{Si}$ steel samples.

Figure 3(b) shows the load-displacement curve of the indentation test on a near-(001) surface of aluminum, where the test was stopped after a single large displacement burst of $\sim 180 \mathrm{~nm}$ using a $1 \mu \mathrm{m}$ spherical indenter. AFM of the residual imprint (Figs. 3(b) (inset) and 3(c)) shows that the pop-in produced a large slip burst on one of the potential slip systems - the one that presumably experienced the largest resolved shear stress during the indentation loading. This leads to the appearance of a step-like feature and an associated pile-up along the [110] direction as shown in Figs. 3(b) and 3(c). The AFM surface profile map shown in Figs. 3(d) and 3(e) indicates that there are major differences in the 
residual surface profile of the indent between the direction of the slip burst and normal to that direction, and that the major changes in the sample surface profile have occurred in the top portion of the residual imprint, which is most likely the region outside the contact region (it should be noted that the profiles measured by AFM shown in Fig. 3 correspond to the unloaded residual imprint and therefore will have changed from the surface profiles under load). Thus, any further loading of the indenter beyond the burst would cause a rapid rise in the contact radius as hypothesized earlier in Fig. 2.

If the indentation is allowed to proceed well beyond the initial burst (Fig. 4(a)), there would be slip bursts along all four of the primary (111) slip planes. Due to the crystallography of the indented (001) surface, the primary slip planes (111) and (111) would intersect the indented plane along the [110] and [110] directions (Figs. 4(a) (inset) and 4(b)). Thus, the activation of the above slip systems would produce an excess material pile-up in these directions resulting in a fourfold pattern of material pile-up around the indent, as has been noted in literature. ${ }^{24}$ With substantial additional indentation beyond the pop-in event, all of asymmetric changes in the surface profile shown earlier in Fig. 3 would be wiped out and the surface profile would essentially be the same as that of a test without the pop-in event.

The insights gained from this combined indentationAFM study regarding the sequence of physical processes occurring during a pop-in event in load-controlled nanoindentation can thus be summarized as follows: (i) During the pop-in event the load, the contact area, and the resultant indentation stress all remain more or less constant. (ii) Immediately following the pop-in, the contact radius increases rapidly over a short regime. This rapid increase in contact radius is due to the changes made to the free surface (just outside of the contact region) as a result of the pop-in event; any further loading thus causes a rapid rise in the contact area. This, in turn, causes the indentation stress to drop sharply subsequent to the pop-in often with an unloading slope that is close to the sample modulus in the case of relatively small to moderate sized pop-ins. (iii) Further loading beyond the pop-in activates several additional smaller popins on all highly stressed slip planes. A consequence of these multitudes of pop-ins is that the memory of the initial pop-in event is eventually wiped out from the indentation response.

This project was initiated by S.P. and S.R.K. at Drexel University with funding from ARO Grant No. W911NF-10-1-0409 (David Stepp, Program Manager). The work was performed, in part, at the Center for Integrated Nanotechnologies, an Office of Science User Facility operated for the U.S. Department of Energy (DOE) Office of Science. Los Alamos National Laboratory, an affirmative action equal opportunity employer, is operated by Los Alamos National Security, LLC, for the National Nuclear Security Administration of the U.S. Department of Energy under Contract No. DE-AC52-06NA25396. S.P. gratefully acknowledges funding from the Los Alamos National Laboratory Director's Postdoctoral Fellowship for this work. S.P. thanks Dr. Manuel Pouchon for allowing the use of the Agilent $\mathrm{G} 200^{\circledR}$ nanoindentation system located at the Paul Scherrer Institut, Villigen, Switzerland, and Dr. Christoph Neiderberger (Empa) for helpful discussions.

${ }^{1}$ T. H. Courtney, Mechanical Behavior of Materials, 2nd ed. (McGraw-Hill Science/Engineering/Math, 1999).

${ }^{2}$ C. A. Schuh, Mater. Today 9(5), 32 (2006).

${ }^{3}$ D. F. Bahr, D. E. Kramer, and W. W. Gerberich, Acta Mater. 46(10), 3605 (1998); Y. L. Chiu and A. H. W. Ngan, ibid. 50(6), 1599 (2002); P. Sudharshan Phani, K. E. Johanns, E. P. George, and G. M. Pharr, J. Mater. Res. 28(19), 2728 (2013).

${ }^{4}$ N. Gane and F. P. Bowden, J. Appl. Phys. 39(3), 1432 (1968).

${ }^{5}$ C. A. Schuh and A. C. Lund, J. Mater. Res. 19(7), 2152 (2004).

${ }^{6}$ S. Suresh, T. G. Nieh, and B. W. Choi, Scr. Mater. 41(9), 951 (1999).

${ }^{7}$ W. W. Gerberich, J. C. Nelson, E. T. Lilleodden, P. Anderson, and J. T. Wyrobek, Acta Mater. 44(9), 3585 (1996); A. Gouldstone, H. J. Koh, K. Y. Zeng, A. E. Giannakopoulos, and S. Suresh, ibid. 48(9), 2277 (2000); A. Gouldstone, K. J. Van Vliet, and S. Suresh, Nature 411(6838), 656 (2001).

${ }^{8}$ A. Kelly and N. H. Macmillan, Strong Solids, 3rd ed. (Clarendon Press, 1986), p. $x i v+423$.

${ }^{9}$ S. Pathak, S. R. Kalidindi, B. Moser, C. Klemenz, and N. Orlovskaya, J. Eur. Ceram. Soc. 28(10), 2039 (2008).

${ }^{10}$ C. A. Schuh and T. G. Nieh, Acta Mater. 51(1), 87 (2003); B. Moser, J. Kuebler, H. Meinhard, W. Muster, and J. Michler, Adv. Eng. Mater. 7(5), 388 (2005).

${ }^{11}$ G. Berces, N. Q. Chinh, A. Juhasz, and J. Lendvai, J. Mater. Res. 13(6), 1411 (1998).

${ }^{12}$ D. Chrobak, K. Nordlund, and R. Nowak, Phys. Rev. Lett. 98(4), 045502 (2007).

${ }^{13}$ V. Domnich, Y. Gogotsi, and S. Dub, Appl. Phys. Lett. 76(16), 2214 (2000).

${ }^{14}$ S. A. Syed Asif and J. B. Pethica, Philos. Mag. A 76(6), 1105 (1997).

${ }^{15}$ See supplementary material at http://dx.doi.org/10.1063/1.4898698 for details of the data analysis protocols used (Eqs. S1 and S2) and for Figs. S1-S3.

${ }^{16}$ T. A. Michalske and J. E. Houston, Acta Mater. 46(2), 391 (1998).

${ }^{17}$ S. Pathak, D. Stojakovic, and S. R. Kalidindi, Acta Mater. 57(10), 3020 (2009).

${ }^{18}$ S. Pathak, D. Stojakovic, R. Doherty, and S. R. Kalidindi, J. Mater. Res. 24(3), 1142 (2009).

${ }^{19}$ S. Pathak, J. Michler, K. Wasmer, and S. R. Kalidindi, J. Mater. Sci. 47(2), 815 (2012).

${ }^{20}$ S. Pathak, J. Shaffer, and S. R. Kalidindi, Scr. Mater. 60(6), 439 (2009).

${ }^{21}$ S. Basu, A. Moseson, and M. W. Barsoum, J. Mater. Res. 21(10), 2628 (2006); J. S. Field and M. V. Swain, ibid. 8(2), 297 (1993); 10(1), 101 (1995).

${ }^{22}$ M. J. Cordill, N. R. Moody, and W. W. Gerberich, J. Mater. Res. 23(6), 1604 (2008); G. M. Pharr, J. H. Strader, and W. C. Oliver, ibid. 24(3), 653 (2009); S. J. Vachhani, R. D. Doherty, and S. R. Kalidindi, Acta Mater. 61(10), 3744 (2013).

${ }^{23}$ S. R. Kalidindi and S. Pathak, Acta Mater. 56(14), 3523 (2008).

${ }^{24}$ Y. Wang, D. Raabe, C. Kluber, and F. Roters, Acta Mater. 52(8), 2229 (2004). 